\title{
Bacteria Colonizing the Ocular Surface in Eyes With Boston Type 1 Keratoprosthesis: Analysis of Biofilm- Forming Capability and Vancomycin Tolerance
}

\author{
Sarmad H. Jassim, ${ }^{1}$ Kavitha R. Sivaraman, ${ }^{1}$ Juan Cristobal Jimenez, ${ }^{2}$ Assraa H. J. Jaboori, ${ }^{1}$ \\ Michael J. Federle, ${ }^{3}$ Jose de la Cruz, ${ }^{1}$ and Maria S. Cortina ${ }^{1}$ \\ ${ }^{1}$ Department of Ophthalmology and Visual Sciences, University of Illinois at Chicago, Chicago, Illinois, United States \\ ${ }^{2}$ Department of Microbiology and Immunology, College of Medicine, University of Illinois at Chicago, Chicago, Illinois, United States \\ ${ }^{3}$ Department of Medicinal Chemistry and Pharmacology, Center for Pharmaceutical Biotechnology, College of Pharmacy, University \\ of Illinois at Chicago, Chicago, Illinois, United States
}

Correspondence: Maria S. Cortina, Department of Ophthalmology and Visual Sciences, University of Illinois at Chicago, $1855 \mathrm{~W}$ Taylor Street, Chicago, IL 60612, USA; mcortina@uic.edu.

SHJ and KRS are joint first authors.

Submitted: April 14, 2015

Accepted: June 5, 2015

Citation: Jassim SH, Sivaraman KR, Jimenez JC, et al. Bacteria colonizing the ocular surface in eyes with Boston type 1 keratoprosthesis: analysis of biofilm-forming capability and vancomycin tolerance. Invest Ophthalmol Vis Sci. 2015;56:4689-4696.

DOI:10.1167/iovs.15-17101
Purpose. To analyze the bacterial microbiota colonizing the ocular surface of patients with Boston type 1 keratoprostheses (K-Pros) for antibacterial resistance patterns and capacity to form biofilms.

Methods. Twenty-seven eyes with a Boston type 1 K-Pro and 16 fellow control eyes from 26 patients were enrolled. The surface of the K-Pro optic and/or the inferior conjunctival fornix was swabbed and plated separately on culture media. Positive cultures were processed to assess for biofilm-forming capability. Microtiter plate adherence assay and polymerase chain reaction for $i c a$ and $a t l E$ genes were used. An in vitro assay of vancomycin tolerance was performed on isolated strains and compared to standard controls with and without biofilmforming capability.

Results. Eighty-five percent of K-Pro eyes and $69 \%$ of control eyes had positive cultures $(P=$ 0.20). All Gram-positive strains exhibited susceptibility to vancomycin by standard testing. Biofilm-forming bacterial isolates were detected in $57.7 \%$ of K-Pro eyes and $53.3 \%$ of control eyes. A vancomycin tolerance assay showed that the antibiotic susceptibility of coagulasenegative staphylococcus (CNS) within biofilms was significant in only three of five biofilmforming strains $(P<0.05)$. In all strains, bacterial cells in planktonic form were more susceptible to vancomycin than in biofilm form $(P<0.001)$.

Conclusions. Coagulase-negative staphylococcus can be isolated from K-Pro surfaces despite the use of vancomycin prophylaxis. In this study, the majority of isolated strains had biofilmforming capability. In vitro vancomycin tolerance assays suggest that biofilm formation decreases susceptibility to vancomycin. This may contribute to higher rates of infectious complications observed in these patients.

Keywords: cornea, keratoprosthesis, biofilm
Since its inception in 1974 , the Boston type 1 keratopros$\checkmark$ thesis (K-Pro) has emerged as a popular option for patients with a poor prognosis for standard keratoplasty. ${ }^{1}$ However, despite the use of topical antibiotic prophylaxis, endophthalmitis and infectious keratitis still occur at a higher rate after keratoprosthesis implantation compared to other types of intraocular surgery. ${ }^{2}$ Factors contributing to the lifelong risk of infection in these patients include a compromised ocular surface, chronic bandage contact lens use, topical steroids, and the presence of a potential space between the K-Pro stem and the donor cornea ${ }^{3,4}$ Additionally, chronic topical antibiotic use may alter the composition of the normal ocular microbiota and generate antibiotic resistance.

A largely unexplored aspect of K-Pro-related infections is the role of biofilms. Biofilms are heterogeneous populations of bacterial cells existing within an extracellular matrix that protects the organism from harsh environments and confers some degree of antibiotic resistance. ${ }^{5,6}$ Biofilms have been detected in several other ophthalmic devices including punctal plugs, nylon sutures, intraocular lenses, and scleral buckles. ${ }^{7-10}$ Additionally, biofilm formation on the surface of contact lenses has been shown to occur after just 48 hours of continuous wear. ${ }^{11}$ Given the high proportion of K-Pro patients using extended-wear bandage contact lenses and the propensity of K-Pro patients to develop ocular infections, the possible contribution of biofilms in eyes with K-Pro is an important area of investigation.

The aim of this current study was to evaluate the biofilmforming capability of bacteria colonizing the ocular surface of patients with the Boston type $1 \mathrm{~K}$-Pro. In addition, we sought to investigate the effect of biofilm formation on vancomycin susceptibility in vitro.

\section{Materials ANd Methods}

This prospective, case-control study was approved by the institutional review board at the University of Illinois Medical Center and adhered to the tenets of the Declaration of Helsinki. 
Patients were enrolled from the cornea subspecialty clinic at the University of Illinois Eye and Ear Infirmary after informed consent was obtained. Inclusion criteria were (1) previously implanted Boston type 1 K-Pro, (2) at least 6 months postoperative follow-up, and (3) use of a bandage contact lens. Eyes with suspected active ocular infections were excluded from the study.

Implantation of Boston type 1 K-Pro was performed by a single surgeon (MSC). All patients were fitted with a bandage contact lens after surgery that was cleaned or replaced at least every 3 months. All bandage contact lenses were soft lenses manufactured by either Kontur (Kontur Kontact Lens, Co., Inc., Hercules, CA, USA) or Air Optix (Alcon, Fort Worth, TX, USA). Preferred antibiotic prophylaxis consisted of topical vancomycin $15 \mathrm{mg} / \mathrm{mL}$ and a topical fourth-generation fluoroquinolone. However, due to variations in insurance drug coverage, regimens differed between patients. Controls were fellow eyes with corneal pathology similar to that in the K-Pro eye in cases of bilateral disease or no corneal pathology in cases of unilateral disease. These eyes were included only if there was no bandage contact lens or topical antibiotic use and no history of ocular surgery for the past 6 months.

In K-Pro eyes, specimens were obtained by swabbing the surface of the K-Pro optic and inferior conjunctival fornix with two separate calcium alginate swabs. In control eyes, only the inferior conjunctival fornix was swabbed. Specimens were then plated separately on standard culture media including blood agar, chocolate agar, and brain-heart infusion. Forniceal swabs were also inoculated in nutrient broth. Antibiotic prophylaxis regimens and all other topical medications being used at the time of culture were recorded. Standard microbiological methods were performed to identify bacterial strains and susceptibility patterns. All strains were studied for susceptibility to erythromycin, gentamycin, oxacilin, levofloxacin, clindamycin, trimethoprim/sulfamethoxazole, vancomycin, tetracycline, and, for some subgroups, rifampicin and ciprofloxacin. All isolated strains were phenotyped according to standard protocols in the clinical microbiology laboratory. Positive cultures were further processed to characterize the biofilm-forming capability of each strain.

\section{Biofilm Detection Methods}

The biofilm-forming capability of a given coagulase-negative staphylococcal isolate was considered positive only if both the following conditions were met: (1) The strain showed phenotypic characteristics of biofilm formation (as demonstrated by a positive microtiter plate assay) and (2) genotypic analysis confirmed the presence of at least one gene known to confer biofilm-forming capability (ica or $a t l E){ }^{12-19}$

Microtiter Plate Assay (MTPA) of Biofilm Growth. Coagulase-negative staphylococcal strains were inoculated from culture plates into Tryptic soy broth (Fisher Scientific, Waltham, MA, USA) and incubated overnight. The following day, $200 \mu \mathrm{L}$ fresh broth was inoculated into flat-bottom 96-well microtiter plates in triplicate with a 1:100 dilution and left to grow in an incubator at $37^{\circ} \mathrm{C}$ overnight. The next day, the media were carefully removed so as not to disturb the congruity of any forming biofilms. Wells were then washed with $200 \mu \mathrm{L} 1 \times$ PBS twice for 5 to 10 minutes. Biofilms were fixed using $200 \mu \mathrm{L}$ Bouin's solution (Sigma Life Science, St. Louis, MO, USA) for 1 hour and then washed twice with PBS. Two hundred microliters Crystal violet $0.2 \%$ was added to the wells for 10 minutes. Stain was removed and washed three times with $1 \times$ PBS. Finally, $200 \mu \mathrm{L} 1 \%$ SDS solution was added to each well. After 10 minutes, the biofilm biomass of the resuspended homogeneous biofilm solution was measured with a microplate reader (BioTek, Winooski, VT, USA) at 570- $\mathrm{nm}$ adsorbance for crystal violet. Standard serial dilution was implemented to avoid overflow readings. Biofilm formation on MTPA was considered positive when the optical density reading on the microplate reader was $>2 .^{12}$

PCR Detection of Biofilm-Associated Genes. Polymerase chain reaction amplification of isolated bacterial colonies was performed according to previously described protocols. ${ }^{17}$ Products were run on $2 \%$ agarose gel electrophoresis stained with ethidium bromide. The reaction was composed of $35 \mu \mathrm{L}$ mastermix solution containing $15 \mu \mathrm{L}$ GoTag Green Master Mix $2 \mathrm{X}, 10 \mu \mathrm{L}$ nuclease-free water (Promega, Madison, WI, USA), 5 $\mu \mathrm{L} i c a$ or atle forward primer, and $5 \mu \mathrm{L} i c a$ or atlE reverse primer. The forward and reverse primers for the $i c a$ gene were 5-TTA TCA ATG CCG CAG TTG TC-3 and 5-GTT TAA CGC GAG TGC GCT AT-3, respectively. The forward and reverse primers used for the atlE gene were 5-CAA CTG CTC AAC CGA GAA CA-3 and 5-TTT GTA GAT GTT GTG CCC CA-3, respectively.

Polymerase chain reaction conditions, including initial denaturation, annealing, and extensions, were performed according to previously described protocols. ${ }^{17}$ For each PCR experiment, Staphylococcus epidermidis strain American Type Culture Collection (ATCC) 35984 (biofilm-forming) was used as a positive control and staphylococcus epidermidis strain ATCC 12228 (nonbiofilm-forming) as negative control. ${ }^{12,20}$ An additional negative control was used that lacked any bacterial colonies.

\section{Vancomycin Susceptibility of Staphylococcal Strains in Planktonic Form and Biofilm Form}

Five representative biofilm-forming CNS strains were selected for a vancomycin tolerance assay. One of these strains was the ATCC positive control (strain A), and the other four were selected from the 29 MTPA-positive isolates. Specifically, strain $\mathrm{B}$ was isolated from eye 14 , strain $\mathrm{C}$ was isolated from eye 18 , and strains $\mathrm{D}$ and $\mathrm{E}$ were two different isolates from eye 21 (Table 1).

For each of the five strains, the susceptibility to vancomycin was determined for the planktonic form versus the biofilm form. Biofilms were grown for each strain in flat-bottom 96well plates as follows: Overnight cultures were inoculated in a 1:100 dilution in a total of $200 \mu \mathrm{L} /$ well of Todd-Hewitt medium supplemented with $0.2 \%$ yeast extract (THY; BD Biosciences, Franklin Lakes, NJ, USA and Amresco, Solon, OH, USA). Plates were incubated for 24 hours at $37^{\circ} \mathrm{C}$, after which time the medium was aspirated and replaced with $100 \mu \mathrm{L}$ either fresh THY (negative control) or THY supplemented with vancomycin at a final concentration of $3.75 \mathrm{mg} / \mathrm{mL}$ (Amresco). The medium was replaced every 24 hours. The experiment ran for a total of 72 hours.

To recover viable bacterial cells from biofilm, the medium was aspirated and the wells were rinsed thoroughly three times with sterile PBS. One hundred microliters of fresh media was added and the bottom of each well was scraped. This suspension was then processed in a sonicator bath to break up any existing biofilms and recover bacterial cells. The planktonic bacterial cells were isolated by plating the media aspirated before every medium exchange on Tryptic Soy Agar plates (Sigma-Aldrich Corp., St. Louis, MO, USA). Serial dilutions of cells were prepared and plated on Tryptic Soy Agar plates. Plates were incubated overnight at $37^{\circ} \mathrm{C}$, and colonies were counted to assess bacterial viability as previously described. ${ }^{21,22}$

\section{Electron Microscopy}

Four contact lenses were obtained from four K-Pro eyes (eyes $14,15,18$, and 24 in Table 1) and sent for electron microscopy. The contact lenses were fixed in $2 \%$ glutaraldehyde and 
TABLE 1. Culture Results From K-Pro and Control Eyes and Biofilm-Forming Capability of Isolated Strains

\begin{tabular}{|c|c|c|c|c|c|}
\hline \multirow{2}{*}{ Case } & \multicolumn{3}{|c|}{ K-Pro Eye } & \multicolumn{2}{|c|}{ Fellow Control Eye } \\
\hline & Antibiotic Prophylaxis & Growth, \pm & Biofilm & Growth, \pm & Biofilm \\
\hline 1 & Vanco & - & N/A & NAC & N/A \\
\hline 2 & Vanco & $+\mathrm{K}$ & + & NAC & N/A \\
\hline 3 & Vanco & - & N/A & NAC & N/A \\
\hline 4 & Vanco & $+\mathrm{F}$ & + & NAC & N/A \\
\hline 5 & Vanco/Gati & $+\mathrm{F}$ & + & - & N/A \\
\hline 6 & Vanco/Gati & $+\mathrm{F}$ & - & NAC & N/A \\
\hline 7 & Vanco & - & N/A & - & N/A \\
\hline 8 & Vanco/Gati & $+\mathrm{F}$ & - & NAC & N/A \\
\hline 9 & Polymyxin & $+\mathrm{F}, \mathrm{K}$ & + & + & + \\
\hline 10 & Vanco & $+\mathrm{F}, \mathrm{K}$ & - & + & - \\
\hline 11 & Vanco & $+\mathrm{F}, \mathrm{K}$ & + & + & - \\
\hline 12 & Vanco & $+\mathrm{F}$ & + & NAC & N/A \\
\hline 13 & Vanco/Gati & $+\mathrm{F}$ & - & - & N/A \\
\hline 14 & Polymyxin & $+\mathrm{F}, \mathrm{K}$ & + & NAC & N/A \\
\hline 15 & Vanco/Gati & $+\mathrm{F}, \mathrm{K}$ & + & NAC & N/A \\
\hline 16 & Vanco/Moxi & $+\mathrm{F}, \mathrm{K}$ & - & + & - \\
\hline 17 & Vanco/Gati & $+\mathrm{F}$ & - & NAC & N/A \\
\hline 18 & Moxi & $+\mathrm{F}$ & + & + & + \\
\hline 19 & Vanco & $+\mathrm{F}$ & - & - & N/A \\
\hline 20 & Vanco & $+\mathrm{F}$ & + & + & + \\
\hline 21 & Vanco & $+\mathrm{F}, \mathrm{K}$ & + & NAC & N/A \\
\hline 22 & Vanco & $+\mathrm{F}, \mathrm{K}$ & + & + & - \\
\hline 23 & Vanco & - & N/A & - & N/A \\
\hline 24 & Moxi & $+\mathrm{F}$ & + & + & - \\
\hline 25 & Vanco & $+\mathrm{F}$ & - & + & + \\
\hline 26 & Vanco/Gati & $+\mathrm{F}$ & - & + & + \\
\hline 27 & Gati & $+\mathrm{F}$ & + & + & - \\
\hline Total & & 27 & 14 & 16 & 5 \\
\hline
\end{tabular}

Vanco, vancomycin; Gati, gatifloxacin; Moxi, moxifloxacin; K, growth observed on culture obtained from keratoprosthesis surface; F, growth observed on culture obtained from conjunctival fornix; NAC, fellow eye not available for culture; N/A, not applicable.

pretreated with $1 \% \mathrm{OsO}_{4}$ (osmium tetroxide) (Electron Microscopy Sciences, Hatfield, PA, USA). The samples were then serially dehydrated through an ascending graded concentration of ethanol, critical-point dried with hexamethyldisilazane (Electron Microscopy Sciences), and then air-dried. Specimens were mounted on aluminum foil stubs with colloidal graphite, sputter coated with a thin film of gold, and examined under a scanning electron microscope (JEOL JSM-6320F Scanning electron microscope, JEOL Ltd., Tokyo, Japan, and Hitachi S3500 scanning electron microscope, Hitachi Ltd, Tokyo, Japan).

\section{Statistical Analysis}

All quantitative parameters for the MTPA and vancomycin tolerance assays were entered into a spreadsheet using Microsoft Excel software (Microsoft, Redmond, WA, USA). Statistical analysis was performed using a Student's $t$-test or $\chi^{2}$ test depending on the sample size of the variable being analyzed.

\section{Results}

\section{Patients and Surveillance Culture Results}

A total of 27 eyes with implanted K-Pros and 16 fellow control eyes from 26 patients were enrolled into the study. Sixteen patients were men and 10 were women. Of the 27 K-Pro eyes, 23 (85\%) showed growth of bacterial isolates compared to 11 of 16 control eyes $(68.8 \% ; P=0.20$; Table 2$)$. The majority of these isolates were coagulase-negative staphylococci (CNS; Table 3). Of the 23 K-Pro eyes with positive cultures, 9 (39.1\%) had isolates directly from the K-Pro surface and 22 (95.7\%) from the fornix. Specifically, 8 K-Pro eyes had positive cultures from both the fornix and front plate, $1 \mathrm{~K}$-Pro eye had a positive culture from only the K-Pro surface, and 14 K-Pro eyes had positive cultures from only the fornix (Table 1; Fig. 1). All isolates from the control eyes were obtained from the inferior fornix.

Among the 23 K-Pro eyes and 11 control eyes with positive cultures, 37 different bacterial strains were isolated from the $\mathrm{K}$ Pro eyes and 11 different strains from the control eyes. Among K-Pro eyes, 16 (69.5\%) showed growth of CNS in isolation, and an additional 5 eyes (21.7\%) grew CNS in combination with other species. In the 5 eyes with mixed culture results, the species isolated in addition to CNS were alpha-hemolytic streptococci (nonpneumococcal), diphtheroids, Propionobacterium acnes, Micrococcus species, Neisseria sicca, and rare anaerobes. All 11 control eyes (100\%) grew only CNS.

Table 3 details the microbiological results of cultures, and Table 1 details the antibiotic prophylaxis regimen for each eye as well as the source and results of the obtained cultures. Notably, of the five eyes with mixed culture results, four were on topical vancomycin prophylaxis at the time the culture was obtained, and the other was on topical polymyxin $\mathrm{B} /$ trimethoprim.

Table 2. Culture Positivity From Swabs of K-Pro Versus Control Eyes

\begin{tabular}{lccc}
\hline & Growth & No Growth & Total \\
\hline K-Pro & 23 & 4 & 27 \\
Control & 11 & 5 & 16 \\
\hline
\end{tabular}



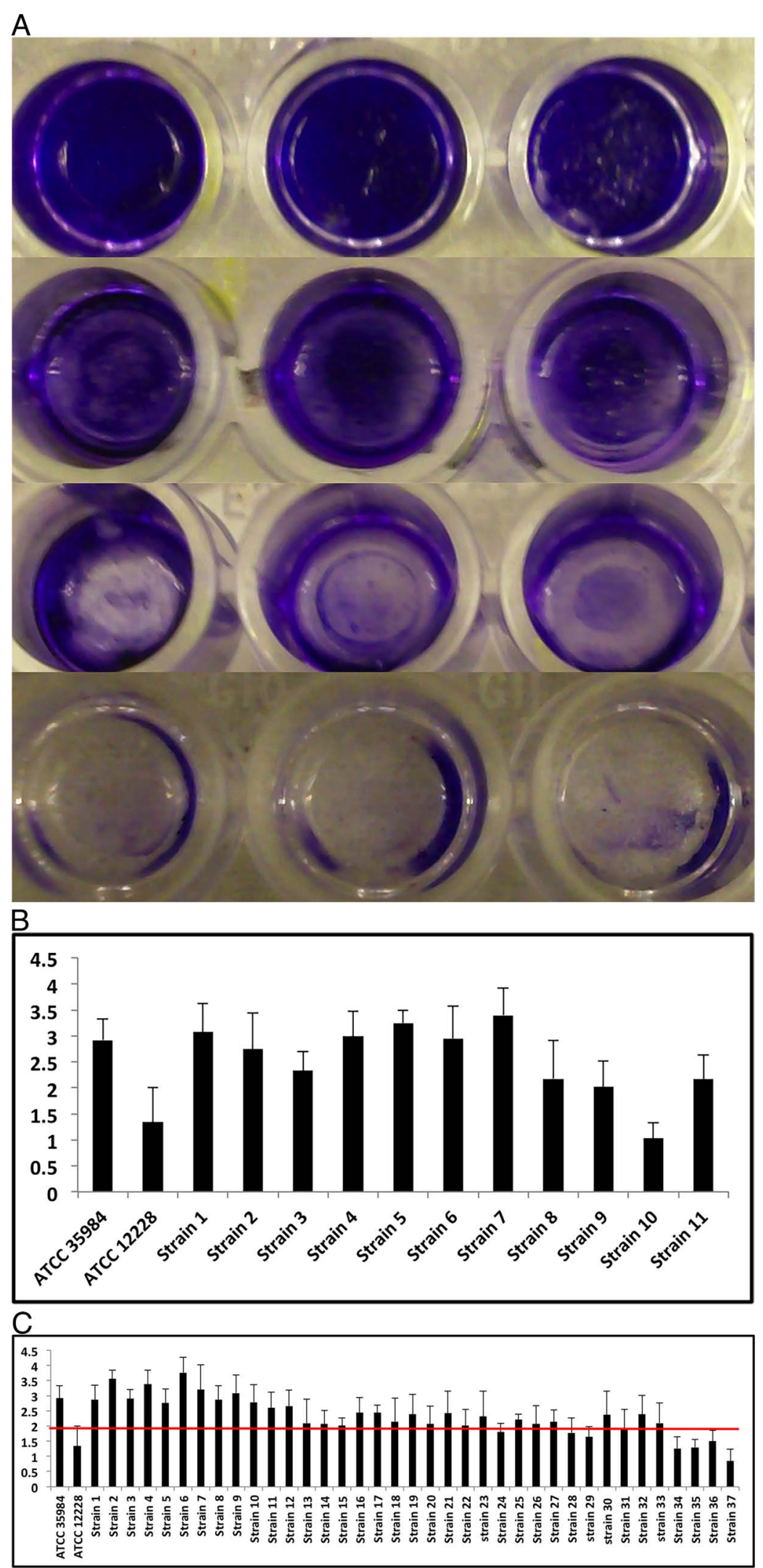

Figure 1. Phenotypic and genotypic characterization of coagulase-negative staphylococci isolated from eyes with an implanted keratoprosthesis. (A) Differential adherence strength of different strains on a microtiter plate assay. The top wells demonstrate strong adherence; the middle wells show mild to moderate adherence; and the bottom wells show nonadherent strains. Biofilm biomass measured by spectrophotometry of 37 coagulase-negative staphylococci strains isolated from K-Pro eyes $(\mathbf{C})$ and 11 strains from control eyes (B) shows that the majority have phenotypic biofilm characteristics with different adherence strength. Strains with an optical density reading $>2$ were considered to be adherent (i.e., biofilmforming), whereas strains with an optical density reading $>2.5$ were considered strongly adherent. ATCC 39584 is the standard biofilm-forming CNS strain used as a positive control. ATCC 12228 is the standard nonbiofilm-forming strain used as a negative control. 
Table 3. Microbiological Results of Positive Surveillance Cultures in K-Pro and Control Eyes

\begin{tabular}{lcc}
\hline & Bacterial Growth & K-Pro, $\boldsymbol{n}=\mathbf{2 3}$ Control, $\boldsymbol{n}=\mathbf{1 1}$ \\
\hline Coagulase-negative staphylococci & $16,69.5 \%$ & $1,4.4 \%$ \\
Diptherioids & $1,4.4 \%$ & - \\
Roseomonas & & - \\
$\begin{array}{l}\text { Mixed: CNS, alpha hemolytic streptococci, diptherioids, Propionibacterium species, Micrococcus } \\
\text { species, } N \text {. sicca, rare anaerobes }\end{array}$ & $5,21.7 \%$ \\
\hline
\end{tabular}

Among all 27 K-Pro eyes enrolled, 22 (81.5\%) were receiving vancomycin prophylaxis alone or in combination with a fourth-generation fluoroquinolone. Of the remaining five eyes (18.5\%), three were on a fourth-generation fluoroquinolone and two were on polymixin $\mathrm{B} /$ trimethoprim.

Of the 23 K-Pro eyes with positive cultures, the single eye with a positive culture from the K-Pro surface was on vancomycin monotherapy. Of the 14 eyes with positive culture from the fornix only, 6 were on a combination of vancomycin and a third-generation fluoroquinolone; three were on a thirdgeneration fluoroquinolone, and 5 were on vancomycin only. Of the 8 eyes with a positive culture from both the fornix and front plate, 2 were on polymixin, 2 others were on a combination of vancomycin and a third-generation fluoroquinolone, and the other 4 were on vancomycin only. There was no difference in the antibiotic resistance patterns between K-Pro and control eyes. All Gram-positive bacterial strains exhibited susceptibility to vancomycin by standard testing, but $22.7 \%$ of isolates were found to be resistant to levofloxacin. No statistically significant difference was observed in bacterial growth between K-Pro and control eyes (Table 2).

\section{Detection of Biofilm-Forming Capability of Coagulase-Negative Staphylococci Isolates}

Microtiter Plate Assay. Of the 37 different coagulasenegative staphylococcal strains isolated from the $23 \mathrm{~K}$-Pro eyes, 29 isolates from 17 eyes were found to be capable of biofilm formation on MTPA. Of the 11 different strains isolated from 11 control eyes with positive cultures, 10 isolates were biofilm formers on MTPA (Fig. 1).

Figure 1A demonstrates the adherence strength of different strains on the bottom of microtiter plates. Biofilm formation on MTPA was considered positive when the optical density reading on the microplate reader was $>2$. Among K-Pro eyes, 29 strongly adherent strains showed readings greater than 2 , and 8 less adherent strains showed readings less than 2 .

PCR Detection of Biofilm-Associated Genes. At least one of the two biofilm-associated genes atlE and ica was detected in isolates from 14 (60.9\%) K-Pro eyes and 5 (45.5\%) control eyes (Table 4). All strains in both the K-Pro and control groups with positive PCR results also had positive biofilm formation on MTPA. Among K-Pro eyes, 7 eyes (9 strains) were positive for $i c a$ and an additional 7 eyes (8 strains) were positive for atle. Among control eyes, 3 were positive for ica and $a t l E$, and 2 were positive for atlE alone.

Three K-Pro eyes and five control eyes did not have evidence of $i c a$ or atlE genes despite positive biofilm formation on MTPA and therefore did not meet our criteria for biofilm formers. No statistically significant difference was observed in the rate of bacterial growth or biofilm formation between $\mathrm{K}$ Pro eyes and control eyes (Tables 2, 4).

\section{Biofilm Vancomycin Tolerance Assay}

Figure 2 illustrates the different survival profile of each of the five selected isolates when exposed to vancomycin in both planktonic and biofilm forms. Planktonic cells of strains A and B died completely after 3 days of vancomycin treatment, whereas the biofilm form of the same strains remained unaffected. Planktonic cells of strains C and D exhibited a rapid decrease in survival, while their respective biofilm forms also showed a 100-fold decrease. Finally, strain E shows an intermediate phenotype in which biofilm cell viability decreases only 10-fold after 3 days of antibiotic treatment. For all strains, the rate of bacterial death was significantly higher for planktonic bacterial cells in comparison to the corresponding biofilm state regardless of the specific strain $(P<0.001)$. Eradication of CNS by vancomycin in its biofilm form was statistically significant only in three of the five biofilm-forming strains studied $(P<0.05)$. In the two other forms (strains A and $B)$, vancomycin was ineffective in eradicating or killing CNS in its biofilm form cells at a statistically significant rate.

\section{Electron Microscopy of Removed Contact Lenses}

Electron microscopy was performed on contact lenses taken from four different K-Pro eyes (Fig. 3). In two lenses (from eyes 15 and 24), bacterial cocci were noted to be grouped in community-like structures and surrounded by extracellular matrix as is morphologically characteristic of a biofilm. In both cases, the biofilms were found on the front surface of the contact lens.

\section{Discussion}

Despite the extraordinary advances made in the field of artificial corneas in the last 50 years, infectious complications remain a significant source of morbidity. Infectious keratitis occurs at a rate of $7.9 \%$ to $17 \%$ in this population, and the risk of K-Pro-associated endophthalmitis has been reported at up to $12 \%{ }^{2,4}$ In an attempt to reduce the incidence of both post-KPro infection and sterile inflammatory melts, two key changes were implemented in the standard postoperative management of K-Pro patients: the introduction of a bandage contact lens and the use of topical antibacterial prophylaxis. ${ }^{23,24}$ More recent studies of K-Pro outcomes in the United States have

TABLE 4. Prevalence of Coagulase-Negative Staphylococci Strains in Surveillance Cultures With Biofilm-Forming Capability as Detected by Polymerase Chain Reaction and Microtiter Plate Adherence Assay

\begin{tabular}{lccc}
\hline & K-Pro Eyes & Control Eyes & $\boldsymbol{P}$ Value \\
\hline Eyes with positive cultures for CNS & $23 / 27,85.2 \%$ & $11 / 16,68.7 \%$ & 0.20 \\
Eyes with biofilm-forming CNS strains & $14 / 23,60.8 \%$ & $5 / 11,45.5 \%$ & 0.40 \\
\hline
\end{tabular}



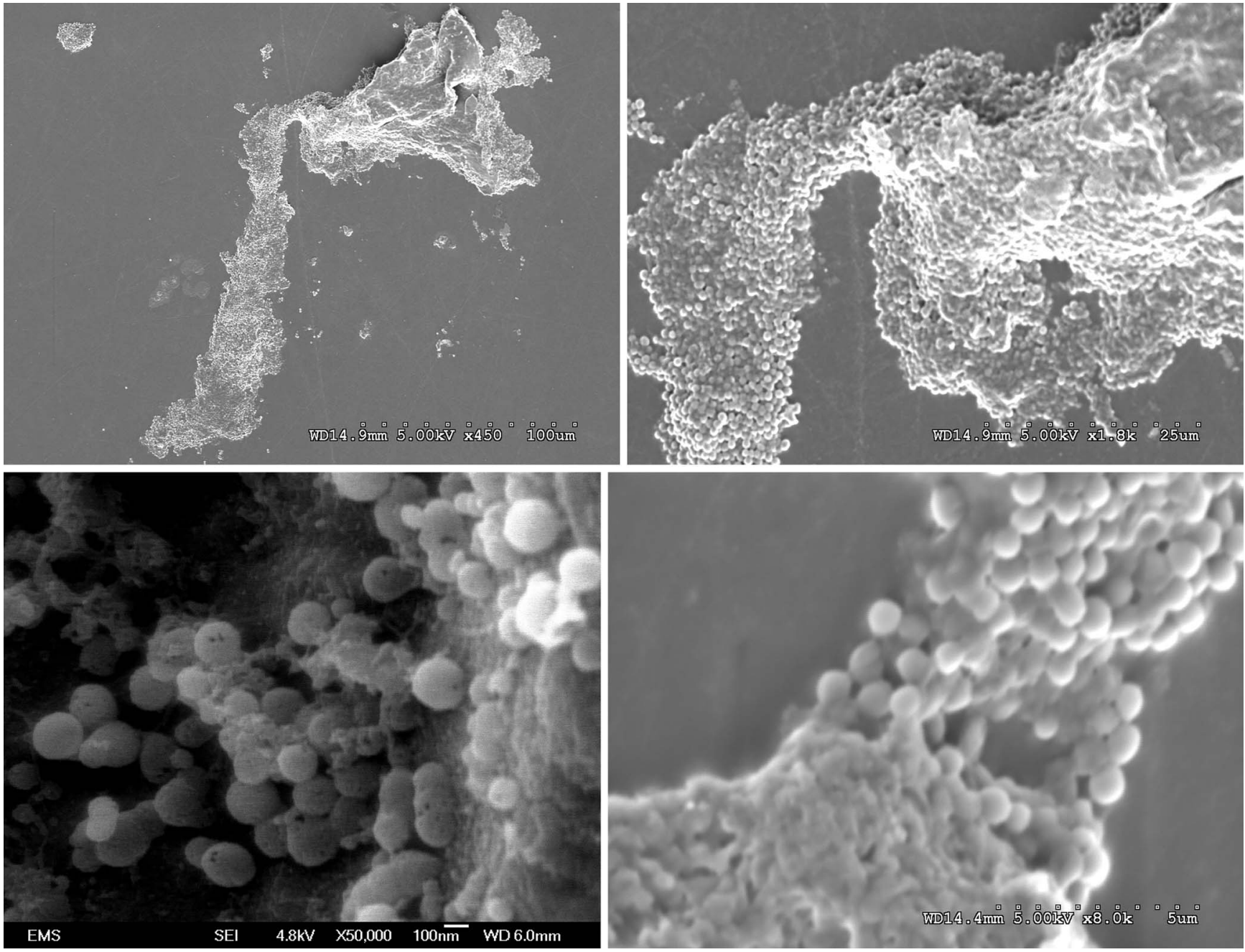

Figure 2. Scanning electron microscope images of the front surfaces of contact lenses from eye 15 (right column) and eye 24 (left column) in Table 2. The top two images at low magnification show adherent sheet-like structures of cellular components. The lower two images at high magnification show cocci-shaped bacteria within the biofilms.

accordingly shown a decrease in the rate of endophthalmitis to approximately $3 \% .^{25}$ Although this represented a major breakthrough in improving K-Pro outcomes, studies of longterm topical antibiotic use show a shift in the ocular surface microbiota, thus raising concerns about increasing antibiotic resistance. ${ }^{26-28}$ Furthermore, the ability of contact lenses to harbor biofilms, which intrinsically increase antimicrobial resistance, has also been well established. ${ }^{29}$

To our knowledge, this is the first study of the biofilm-forming capabilities of ocular surface microbiota in K-Pro patients. Although our study found no difference in the incidence of positive cultures between K-Pro and control eyes, the control eyes with positive cultures grew only CNS. By contrast, K-Pro eyes grew CNS, a wide variety of other Gram-positive organisms, and Roseomonas-a Gram-negative coccobacillus that has only very rarely been implicated as the causative agent in ocular infections. ${ }^{30}$ Although the use of topical antibiotics did not increase the rate of culture positivity in K-Pro patients, the range of organisms colonizing the ocular surface does appear to be altered. None of the eyes in our study experienced infectious complications during the period of investigation, but prior studies have suggested that the normal ocular surface micribiota play a protective role in preventing proliferation of pathogenic species. ${ }^{31}$ Alterations in the composition of the normal bacterial microbiota may therefore predispose to ocular surface infection, such as bacterial or fungal keratitis.

Interestingly, although K-Pro eyes and control eyes differed in the composition of colonizing organisms, all Gram-positive organisms in both groups were sensitive to vancomycin. This supports the continued use of topical vancomycin prophylaxis to prevent Gram-positive infections; however, as noted above, there may be a shift toward an increased proportion of Gram-negative colonizing bacteria. As nearly one-quarter of bacterial isolates were resistant to levofloxacin, this suggests that vancomycin in addition to another broad-spectrum antibiotic agent (such as polymixin B-trimethoprim) may theoretically be more effective.

One limitation of in vitro antimicrobial susceptibility testing is that standard methods do not differentiate between planktonic (free-floating) bacteria and those that exist in biofilms. All strains of CNS in our study were deemed susceptible to vancomycin by standard sensitivity testing. However, when the four biofilm-forming strains and one additional positive control were tested in both planktonic and biofilm forms, the efficacy of vancomycin was significantly diminished against biofilm in all cases. Although CNS are normally commensal organisms, they have been implicated in as many as $83 \%$ of culture-proven keratitis cases and $16 \%$ of endophthalmitis cases in eyes with Boston type $1 \mathrm{~K}$-Pros. ${ }^{5,32,33}$ Our results suggest that in situations in which coagulase 

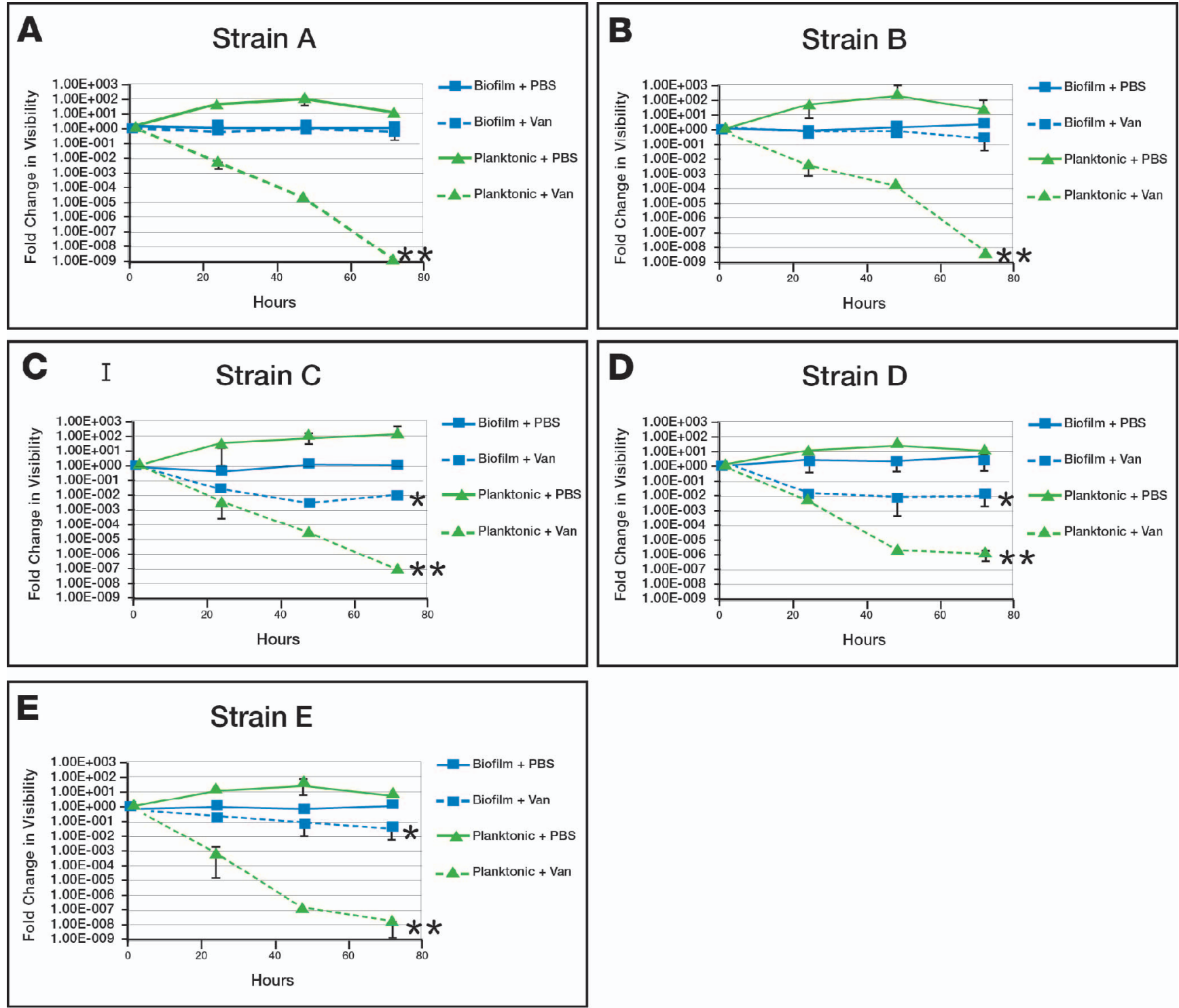

Figure 3. Vancomycin tolerance assay. All parts of the figure show the efficacy of vancomycin in eradicating coagulase-negative staphylococci (CNS) in both biofilm form and planktonic forms over time. All strains in this figure were capable of biofilm formation. (A) and (B), which represent strains A and B, respectively, show that vancomycin was ineffective in eradicating or killing CNS biofilm cells at a statistically significant rate. (C), (D), and (E) demonstrate that vancomycin was effective in killing CNS in biofilm form in strains C, D, and E, respectively. For all strains, vancomycin had higher efficacy in killing the planktonic form of the bacteria versus the biofilm form of the same strain $(P<0.05)$. Van, vancomycin; PBS, phosphate-buffered saline.

negative staphylococci become pathogenic, vancomycin may be substantially less effective in controlling an infection if the bacteria exist within a biofilm matrix.

In our study, both K-Pro and control eyes harbored CNS isolates capable of forming biofilms. One limitation of our investigation is that we are unable to determine under what circumstances and at what rate these bacteria actually form a biofilm. However, our scanning electron microscopy images of bacterial biofilms on bandage contact lenses from K-Pro eyes support that these synthetic surfaces provide a substrate for biofilm formation as has been demonstrated in the contact lens literature previously. ${ }^{34}$ Although bandage contact lens use is important for preventing sterile corneal melts in eyes with $\mathrm{K}$ Pro, ${ }^{23}$ biofilm formation has been linked to an increased risk of keratitis in soft contact lens wearers with otherwise healthy eyes. In addition to the risks associated with soft contact lenses, K-Pro patients have an additional synthetic surface with the K-Pro optic and are frequently also on maintenance topical steroids. This argues for frequent replacement of soft contact lenses in K-Pro patients, and possibly frequent cleansing of the contact and ocular surface in between physician visits.

As contact lenses are likely to remain a mainstay of postoperative K-Pro management, future research should focus on how to improve the penetration of otherwise effective antibiotics into bacterial biofilms. We also recommend that clinicians be vigilant about frequent contact lens replacement and ocular surface cleansing, although studies have yet to validate the efficacy of these measures to reduce the incidence of microbial keratitis and other infectious K-Pro complications.

\section{Acknowledgments}

Supported in part by a departmental grant from Research to Prevent Blindness. 
Disclosure: S.H. Jassim, None; K.R. Sivaraman, None; J.C. Jimenez, None; A.H.J. Jaboori, None; M.J. Federle, None; J. de la Cruz, None; S.M. Cortina, None

\section{References}

1. Dohlman C, Schneider M, Doane M. Prosthokeratoplasty. Am J Ophthalmol. 1974;77:694-700.

2. Nouri M, Terada H, Alfonso E, et al. Endophthalmitis after keratoprosthesis: incidence, bacterial causes, and risk factors. Arch Ophthalmol. 2001;119:484-489.

3. Chan C, Holland E. Infectious keratitis after Boston type 1 keratoprosthesis implantation. Cornea. 2012;31:1128-1134.

4. Chan CC, Holland EJ. Infectious endophthalmitis after Boston type 1 keratoprosthesis implantation. Cornea. 2012;31:346349.

5. Robertson DM, Parks QM, Young RL, et al. Disruption of contact lens-associated Pseudomonas aeruginosa biofilms formed in the presence of neutrophils. Invest Ophthalmol Vis Sci. 2011;52:2844-2850.

6. Zegans ME, Becker HI, Budzik J, et al. The role of bacterial biofilms in ocular infections. DNA Cell Biol. 2002;21:415-420.

7. Behlau I, Gilmore M. Microbial biofilms in ophthalmology and infectious disease. Arch Ophthalmol. 2008;126:1572-1581.

8. Sugita J, Yokoi N, Fullwood NJ, et al. The detection of bacteria and bacterial biofilms in punctal plug holes. Cornea. 2001;20: 362-365.

9. Pan JC, Lau BH, Fam HB, et al. Evaluation of biofilm formation on nylon sutures removed from clinically noninfected eyes after cataract surgery. J Cataract Refract Surg. 2004;30:19721976.

10. Miller KV, Eisley KM, Shanks RM, et al. Recurrent enterococcal endophthalmitis seeded by an intraocular lens biofilm. $J$ Cataract Refract Surg. 2011;37:1355-1359.

11. Elder M, Stapleton F, Evans E, et al. Biofilm-related infections in ophthalmology. Eye. 1995;9:102-109.

12. Paluch-Oles J, Magrys A, Koziol-Montewka M, et al. The phenotypic and genetic biofilm formation characteristics of coagulase-negative staphylococci isolates in children with otitis media. Int J Pediatr Otorhinolaryngol. 2011;75:126130.

13. Makki AR, Sharma S, Duggirala A, et al. Phenotypic and genotypic characterization of coagulase negative staphylococci (CoNS) other than Staphylococcus epidermidis isolated from ocular infections. Invest Ophthalmol Vis Sci. 2011;52: 9018-9022.

14. Sharma P, Lahiri KK, Kapila K. Conventional and molecular characterization of coagulase-negative staphylococcus in hospital isolates. Indian J Pathol Microbiol. 2011;54:85-89.

15. Arciola CR, Gamberini S, Campoccia D, et al. A multiplex PCR method for the detection of all five individual genes of ica locus in Staphylococcus epidermidis. A survey on 400 clinical isolates from prosthesis-associated infections. J Biomed Mater Res A. 2005;75:408-413.

16. Cue D, Lei MG, Lee CY. Genetic regulation of the intercellular adhesion locus in staphylococci. Front Cell Infect Microbiol. 2012;2:38.
17. Patel JD, Colton E, Ebert M, et al. Gene expression during S. epidermidis biofilm formation on biomaterials. J Biomed Mater Res A. 2012;100:2863-2869.

18. Frebourg NB, Lefebvre S, Baert S, et al. PCR-Based assay for discrimination between invasive and contaminating Staphylococcus epidermidis strains. J Clin Microbiol. 2000;38:877880.

19. Iorio NL, Lopes AP, Schuenck RP, et al. A combination of methods to evaluate biofilm production may help to determine the clinical relevance of Staphylococcus in blood cultures. Microbiol Immunol. 2011;55:28-33.

20. Christensen GD, Barker LP, Mawhinney TP, et al. Identification of an antigenic marker of slime production for Staphylococcus epidermidis. Infect Immun. 1990;58:2906-2911.

21. Smith K, Gemmell CG, Lang S. Telavancin shows superior activity to vancomycin with multidrug-resistant Staphylococcus aureus in a range of in vitro biofilm models. Eur J Clin Microbiol Infect Dis. 2013;32:1327-1332.

22. Cerca N, Martins S, Cerca F, et al. Comparative assessment of antibiotic susceptibility of coagulase-negative staphylococci in biofilm versus planktonic culture as assessed by bacterial enumeration or rapid XTT colorimetry. J Antimicrob Chemother. 2005;56:331-336.

23. Harissi-Dagher $\mathrm{M}$, Beyer $\mathrm{J}$, Dohlman $\mathrm{CH}$. The role of soft contact lenses as an adjunct to the Boston keratoprosthesis. Int Ophthalmol Clin. 2008;48:43-51.

24. Durand M, Dohlman CH. Successful prevention of bacterial endophthalmitis in eyes with the Boston keratoprosthesis. Cornea. 2009;28:896-901.

25. Behlau I, Martin KV, Martin JN, et al. Infectious endophthalmitis in Boston keratoprosthesis: incidence and prevention. Acta Ophthalmol. 2014;92:e546-e555.

26. Dave SB, Toma HS, Kim SJ. Changes in ocular flora in eyes exposed to ophthalmic antibiotics. Ophthalmology. 2013;120: 937-941.

27. Yin VT, Weisbrod DJ, Eng KT, et al. Antibiotic resistance of ocular surface flora with repeated use of a topical antibiotic after intravitreal injection. JAMA Ophthalmol. 2013;131:456461.

28. Robert MC, Eid EP, Saint-Antoine P, et al. Microbial colonization and antibacterial resistance patterns after Boston type 1 keratoprosthesis. Opbthalmology. 2013;120:1521-1528.

29. Szczotka-Flynn LB, Imamura Y, Chandra J, et al. Increased resistance of contact lens-related bacterial biofilms to antimicrobial activity of soft contact lens care solutions. Cornea. 2009;28:918-926.

30. Goyal S, Warner DB. Roseomonas keratitis after remote penetrating keratoplasty. Graefes Arch Clin Exp Ophthalmol. 2013;251:1025-1027.

31. McClellan KA. Mucosal defense of the outer eye. Surv Ophthalmol. 1997;42:233-246.

32. Kim MJ, Yu F, Aldave AJ. Microbial keratitis after Boston type I keratoprosthesis implantation: incidence, organisms, risk factors, and outcomes. Ophthalmology. 2013;120:2209-2216.

33. Robert MC, Moussally K, Harissi-Dagher M. Review of endophthalmitis following Boston keratoprosthesis type 1 . Br J Ophthalmol. 2012;96:776-780.

34. Willcox MD. Microbial adhesion to silicone hydrogel lenses: a review. Eye Contact Lens. 2013;39:61-66. 